\title{
La depresión es un evento frecuente en los médicos residentes
}

\section{Depression is frequent event among resident physicians}

\section{Objetivos}

Estimar la prevalencia de depresión o síntomas depresivos en médicos residentes.

\section{Diseño}

Revisión sistemática y meta análisis.

\section{Fuente de datos}

Se revisaron EMBASE, ERIC, MEDLINE y PsycINFO limitando la búsqueda a estudios publicados entre 1963 y 2015

\section{Selección de estudios}

Se incluyeron estudios publicados en revistas con revisión por pares, que utilizaran un método validado para evaluar depresión o síntomas depresivos.

\section{Extracción de datos}

Dos investigadores independientes extrajeron información sobre
Mata DA. JAMA. 2015 Dec 8;314(22):2373-83. las características de los estudios y los resultados. La prevalencia de depresión o síntomas depresivos se calculó agrupando las estimaciones de cada estudio utilizando un modelo de efectos aleatorios.

\section{Resultados principales}

Se incluyeron 31 estudios de corte transversal y 23 longitudinales (17.560 individuos). Tres estudios utilizaron entrevistas clínicas y 51 estudios instrumentos de auto-reporte. La prevalencia global agrupada de depresión o síntomas depresivos fue de $28,8 \%$ (IC $95 \%$ 25,3 a $32,5 \%$ ), con evidencia significativa de heterogeneidad entre los estudios (I2=95.8\%, p<0,001). La prevalencia de depresión según la escala utilizada se resume en la tabla 1.

En el análisis secundario de siete estudios longitudinales, el incremento promedio de síntomas depresivos al inicio de la residencia fue de 15,8\% (rango 0,3 a 26,3\%; RR 4,5; $p<0,05$ ).

Tabla 1. Prevalencia de depresión según la escala utilizada.

\begin{tabular}{c|c|c|c|c} 
Instrumento & Estudios & Positivos & Total & Prevalencia \% (IC 95\%) \\
\hline CES-D & 7 & 2.111 & 8.113 & $28,4(24,2 \mathrm{a} 33,0)$ \\
\hline BDI (21 ítems) & 7 & 254 & 894 & $26,6(12,9 \mathrm{a} 47,1)$ \\
\hline PRIME-MD & 8 & 1.349 & 2.891 & $43,2(37,6 \mathrm{a} 49,0)$ \\
\hline PHQ-9 & 4 & 741 & 3.577 & $20,9(17,5 \mathrm{a} 24,7)$ \\
\hline Zung SDS & 2 & 159 & 382 & $30,4(8,6 \mathrm{a} 67,1)$ \\
\hline HANDS & 3 & 363 & 1.716 & $21,2(19,3-23,2)$ \\
\hline
\end{tabular}

\section{Conclusiones}

La prevalencia estimada fue de $28,8 \%$, variando entre $20,9 \%$ y $43,2 \%$ dependiendo del instrumento utilizado.
Fuente de financiamiento/conflicto de interés de los autores: US Department of State, National Institute of Health.

\section{Comentario}

La depresión en los médicos residentes se asocia a cuidados de menor calidad y aumento en los errores médicos ${ }^{1,2}$. A causa de la heterogeneidad en cuanto a los instrumentos utilizados y las poblaciones estudiadas, la prevalencia de depresión mayor no pudo ser efectivamente estimada. Sin embargo, un análisis secundario utilizando escalas validadas de alta especificidad (>88\%) arrojó una prevalencia de $20,2 \%$, lo cual podría reflejar mejor el verdadero impacto de este problema durante la residencia.

\section{Conclusiones del comentador}

El maltrato, la precarización laboral, y las semanas laborales de más de 100 horas no son una experiencia de aprendizaje y sin embargo, no existe ninguna residencia que no posea al menos una de estas tres características. La depresión en médicos residentes es síntoma de un modelo educativo agotado, que se sigue sosteniendo por sus beneficios en la reducción de costos y la ausencia de propuestas alternativas.

Agustín González Calbano [ Médico de familia de la Unidad Sanitaria Barrio Saladero - Secretaría de Salud de Bahía Blanca agustin.gonzalez@uns.edu.ar ]

Gonzalez Calbano A La depresión es un evento frecuente en los médicos residentes. Evid Act Pract Ambul. 2016;19(2):49. Abr-Jun. Comentado de: Mata DA, y col. Prevalence of Depression and Depressive Symptoms Among Resident Physicians: A Systematic Review and Meta-analysis. JAMA. 2015;314(22):2373-83. PMID: 26647259.

\section{Referencias:}

1. Landrigan $\mathrm{CP}$, y col. Effect of reducing interns' work hours on serious medical errors in intensive care units. N Engl J Med 2004;351:1838-48.

2. Barger LK, y col. Impact of Extended-Duration Shifts on Medical Errors, Adverse Events, and Attentional Failures. PLoS Med 2006;3(12):e487. doi:10.1371/joumal.pmed.0030487. 